\title{
Profesorado universitario y educación inclusiva: respondiendo a sus necesidades de formación
}

\author{
Anabel Moriña \\ Universidade de Sevilla - Sevilla - Espanha \\ Rafael Carballo \\ Universidade de Sevilla - Sevilla - Espanha \\ Universidade Isabel I - Burgos - Espanha
}

\begin{abstract}
Resumen
Este artículo presenta los resultados de una investigación cuyo objetivo era diseñar, desarrollar y evaluar un programa de formación sobre educación inclusiva y discapacidad dirigido al profesorado universitario. Mediante el uso de una metodología cualitativa, se realizó la evaluación en tres momentos: una evaluación inicial de las necesidades de formación de los participantes; una evaluación de proceso durante el desarrollo del programa; y una evaluación final de los resultados una vez concluida la formación. Los instrumentos empleados para la recogida de datos fueron la entrevista semi-estructurada individual y grupal, el cuestionario abierto y la observación. Este trabajo ofrece una visión sobre las principales necesidades de formación de los 20 participantes y cómo el programa da respuesta a éstas, exponiendo los aprendizajes más relevantes adquiridos por los docentes y contrastándolos con la situación de partida. Finalmente, en las conclusiones se expone una discusión de los resultados con otras investigaciones previas.
\end{abstract}

Palabras Clave: Formación de profesores; evaluación de programa; educación inclusiva.

\section{University teaching staff and inclusive education: responding to their training needs}

\begin{abstract}
This article presents the results of a research whose objective was to design, develop and evaluate a training program on inclusive education and disability aimed at university teaching staff. Through the use of a qualitative methodology, the evaluation was carried out in three moments: an initial evaluation of the training needs of the participants; a process evaluation during the development of the program; and a final evaluation of the results once the training is completed. The instruments used for the data collection were the semi-structured individual and group interview, the open questionnaire and the observation. This work offers a vision about the main training needs of the 20 participants and how the program responds to them, exposing the most relevant learning acquired by the teachers and contrasting them with the starting situation. Finally, the conclusions show a discussion of the results of other previous investigations.
\end{abstract}

Keywords: Teacher training; program evaluation; inclusive education.

\section{Professorado universitário e educação inclusiva: respondendo a suas necessidades de formação}

\begin{abstract}
Resumo
Este artigo apresenta os resultados de uma pesquisa cujo objetivo era conceber, desenvolver e avaliar um programa de formação sobre educação inclusiva e deficiência dirigido aos professores universitários. Mediante o uso de uma metodologia qualitativa, realizou-se a avaliação em três momentos: avaliação inicial das necessidades de formação dos participantes; avaliação do processo durante o desenvolvimento do programa e avaliação final dos resultados uma vez concluída a formação. Os instrumentos empregados para a coleta de dados foram a entrevista semiestruturada individual e grupal, o questionário aberto e a observação. Este estudo oferece uma visão sobre as principais necessidades de formação dos 20 participantes e como o programa da resposta a estas, expondo as aprendizagens mais relevantes adquiridos pelos docentes e confrontando-as com a situação de inicial. Finalmente, nas conclusões se expõe uma discussão dos resultados com outras pesquisas prévias.

Palavras-chave: Formação de professores; avaliação de programa; educação inclusiva.
\end{abstract}




\section{Introducción}

Las barreras y ayudas que los estudiantes con discapacidad identifican en su paso por la universidad han sido ampliamente documentada en la literatura científica (Bell, Devecchi, Guckin, \& Shevlin, 2017; Fuller, Healey, Bradley,\& Hall, 2004; Mullins \& Preyde, 2013). En la actualidad, la mayoría de universidades cuentan con servicios de apoyo y normativas que regulan los derechos de las personas con discapacidad, aunque estas no son suficientes. La enseñanza superior, por tanto, se enfrenta al desafío de desarrollar procesos educativos inclusivos que permitan el aprendizaje, participación y pertenencia de todo el alumnado (Hardy \& Woodcock, 2015; Messiou \& cols., 2016; Moriña, 2017).

En las investigaciones desarrolladas sobre las barreras del alumnado con discapacidad, éste identifica como el principal obstáculo al profesorado, en cuanto a actitudes negativas y falta de formación para atender sus necesidades (Garrison-Wade, 2012; Moswela \& Mukhopadhyay, 2011; Stein, 2014; Strnadová, Hájková, \& Kvetonová, 2015). Los estudiantes indican que hay profesores que no están predispuestos a realizar los ajustes razonables recogidos en la normativa (Fuller \& cols., 2004; Yssel, Pak, \& Beilke, 2016). El profesor, según relata el alumnado, no modifica aspectos como la metodología o la evaluación (Bessant, 2012). La mayoría de trabajos coinciden en señalar que el profesorado necesita estar informado, formado y sensibilizado para atender adecuadamente a sus necesidades (Milic \& Dowling, 2015; Vickerman \& Blundell, 2010).

En los estudios sobre el profesorado, se señala su limitada experiencia y una mínima formación en discapacidad y en prácticas educativas inclusivas (Black, Weinberg, \& Brodwin, 2014; Cook, Rumrill, \& Tankersley, 2009).

En España, al igual que en la mayoría de países, la formación del profesorado universitario es voluntaria y gratuita y para enseñar no se requiere recibir formación pedagógica (Carballo, 2016; Gunersel \& Etienne, 2014). No obstante, se cuenta con algunas experiencias que han sido desarrolladas. Por ejemplo, el proyecto "Teachability" en Escocia (Simpson, 2002) provee un recurso para facilitar una revisión de la enseñanza y aprendizaje para los académicos, con la idea de mejorar la accesibilidad. En Inglaterra, Hockings, Brett y Terentjevs (2012) ofrecen un repositorio virtual de videos en aulas reales que muestran prácticas de enseñanza y aprendizaje inclusivas. En Irlanda, Griffiths (2010) ha desarrollado una guía para que la enseñanza sea inclusiva. Y en España, se cuenta con algunas experiencias de diseño de programas formativos para profesores universitarios (Dotras, Llinares, \& López, 2008; Moriña, 2018).

La mayoría de las iniciativas formativas se han quedado solo en el diseño y no han sido evaluados su desarrollo e impacto. Algunos de los trabajos que sí han sido evaluados coinciden en que cuando los profesores se forman en discapacidad, inclusión y Diseño Universal de Aprendizaje (DUA), repercute positivamente en los estudiantes, con o sin discapacidad (Cunningham, 2013; Garrison-Wade, 2012; Redpath \& cols., 2013). En diversos trabajos se concluye que la formación recibida contribuyó a mejorar el conocimiento y sensibilidad hacia estudiantes con discapacidad y las actitudes del profesorado (Davies, Schelly \& Spooner, 2013; Lombardi, Murray, \& Gerdes, 2011; Murray, Lombardi \& Wren, 2011).

Respecto a los contenidos en la formación, el profesorado considera que es importante conocer sus obligaciones legales, técnicas en el diseño del currículo, ajustes en la enseñanza, información sobre recursos disponibles para estudiantes con discapacidad, uso efectivo de prácticas instruccionales, conocimientos de las características de la discapacidad o información sobre cómo acceder a los servicios de los estudiantes (Cook \& cols., 2009; Gelbar, Madaus, Lombardi, Faggella-Luby, \& Dukes, 2015).

Además, se precisa diseñar programas de formación que sean desarrollados y evaluados para comprobar si realmente son efectivos y si contribuyen a que el profesorado comprenda la importancia de dar una respuesta inclusiva al alumnado con discapacidad y sepa cómo hacerlo en la práctica.

Teniendo en cuenta estos antecedentes, este artículo se centra en analizar las necesidades de formación del profesorado universitario en relación a la educación inclusiva y la discapacidad, así como los resultados de aprendizaje de un programa de formación que da respuesta a las necesidades iniciales en relación a la atención al alumnado con discapacidad.

\section{Método}

Este trabajo forma parte de una investigación más amplia financiada por el Ministerio de Economía y Competitividad de España: "Caminando hacia la inclusión social y educativa en la universidad" (Ref. EDU2013-46303-R/ fondos FEDER). Durante los cuatro años de duración del proyecto (2014-2017) se pretendía formar al profesorado en dar una respuesta inclusiva a las necesidades de los estudiantes con discapacidad.

Se realizó una evaluación cualitativa del programa de formación que tuvo lugar en tres momentos: antes de diseñar el programa (evaluación de las necesidades de formación); durante el desarrollo del programa (evaluación del proceso de formación); y al finalizar la formación (evaluación de los resultados y el impacto de la formación).

Este artículo se centra, de manera más concreta, en los resultados de las evaluaciones realizadas al inicio y al final de todo el proceso, en las que se pretendían conocer las necesidades formativas iniciales y qué aprendieron con el programa, respectivamente.

\section{El programa de formación}

"Caminando hacia la inclusión social y educativa en la universidad" es un programa de formación dirigido al profesorado que pretendía capacitarlos para ofrecer una respuesta 
educativa inclusiva al alumnado con discapacidad. El programa, diseñado por el equipo de investigación, se desarrolló a través de la modalidad de formación blended-learning. La duración de la formación fue de seis meses (enero-junio de 2016), con un total de 54 horas (12 horas presenciales y 42 online).

La formación presencial se organizó en tres sesiones de cuatro horas, al inicio, en la mitad y al final de la formación. En estas sesiones se invitaba a estudiantes con discapacidad para que en primera persona contaran sus experiencias en la universidad y se resolvían dudas de la formación online.

A través de la plataforma BlackBoard, la parte online se basó en el desarrollo de módulos de aprendizaje con contenidos teóricos y prácticos y actividades para aplicar los contenidos y que permitían interactuar en foros de discusión.

Los contenidos del programa estaban organizados en torno a ocho módulos que abordaban el concepto de discapacidad, el modelo social de discapacidad y educación inclusiva; barreras y ayudas identificadas por universitarios con discapacidad; normativa universitaria y servicios de apoyo; diferentes tipos de discapacidad, necesidades y principales modificaciones del currículo; y por último, Diseño Universal de Aprendizaje.

\section{Participantes en la Formación}

El contacto con la muestra se estableció a través de la publicitación del programa por medio del centro de formación de la universidad, ofertando 30 plazas para la participación en la formación. Finalmente fueron 20 los profesores que participaron y finalizaron el programa, ya que, en un momento inicial se contó con 23 inscripciones, pero dos docentes no iniciaron el curso y otro de ellos abandonó poco después de dar comienzo.

Seis criterios para seleccionar la muestra se explicitaron en la publicidad del curso: profesorado de todos los campos de conocimiento; profesores de ambos sexos; diferencias en cuanto a los años de experiencia en la universidad; experiencia con estudiantes con discapacidad, compromiso de introducir cambios en las aulas; disponibilidad para participar activamente. Todos los criterios pudieron ser cumplidos a excepción de la experiencia con estudiantes con discapacidad, ya que seis profesores nunca habían enseñado a estudiantes con discapacidad; y la diversidad de campos de conocimiento, puesto que ningún profesor de Ciencias Experimentales y Enseñanzas Técnicas estuvo interesado en realizar el curso.

En cuanto a los perfiles de los docentes, 12 pertenecían al área de Ciencias Sociales y Jurídicas (ocho de ellos provinientes de la Facultad de Ciencias de la Educación), cuatro de Ciencias de la Salud y otros cuatro de Humanidades. En relación al sexo, la mayoría, 12 docentes, eran mujeres y los ocho restantes eran hombres. Por otra parte, seis de los participantes nunca habían tenido ninguna experiencia con alumnado con discapacidad en el aula. Por último, la mitad de los participantes contaban con más de cinco años de experiencia docente, mientras que el resto tenía una trayectoria más breve.

\section{Diseño e instrumentos de evaluación}

Se realizó una evaluación cualitativa del programa de formación en los tres momentos previamente descritos. Los instrumentos empleados para la recogida de información fueron entrevistas semi-estructuradas grupales e individuales, cuestionarios escritos abiertos y observación de las sesiones presenciales.

Tanto en la evaluación inicial como en la final se realizaron tres entrevistas grupales de 1 hora 30 minutos aproximados cada una. A cuatro profesores, que no pudieron participar en las entrevistas grupales finales, se les realizaron entrevistas individuales con una duración de 1 hora. Toda la información ofrecida por los participantes fue grabada en audio y posteriormente transcrita. Además, cada uno de los participantes cumplimentó un cuestionario con preguntas abiertas en el que reflexionaba por escrito sobre sus necesidades de formación (cuestionario de evaluación inicial) y sobre los aprendizajes del curso (cuestionario de evaluación final). Por último, la observación fue realizada por dos miembros del equipo de investigación en las sesiones presenciales de la formación.

\section{Análisis de Datos}

Los datos fueron analizados por medio de un sistema de categorías y códigos, que se generó en el equipo de investigación de forma inductiva, siguiendo la propuesta de Miles y Huberman (1994). A través de éste se realizó un análisis comparativo con toda la información recogida, apoyado por el uso del programa informático MaxQDA12.

\section{Cuestiones Éticas}

Se aseguró desde el inicio que los datos recogidos se tratarían de forma anónima y confidencial. Se informó a todos los participantes de que esos datos serían destruidos y excluidos del informe de investigación en caso de que no quisiera continuar participando.

\section{Resultados}

Los resultados que a continuación se presentan están organizados en torno a tres áreas temáticas: la importancia de formación en educación inclusiva y su efecto en la sensibilidad del profesorado; la necesidad de información sobre el alumnado con discapacidad; y los conocimientos prácticos más relevantes en los docentes deben adquirir. En cada uno de ellos se presenta, en primer lugar, la situación 
de partida de los participantes antes de iniciar el programa $y$, seguidamente, los resultados finales tras la formación y cómo ésta da respuesta a sus necesidades.

\section{¿Por qué formarse en educación inclusiva?}

Los participantes en este estudio consideraban que la situación a la que se enfrentaban cuando en el aula había un estudiante con una discapacidad no era fácil, máxime cuando no se contaba con una preparación previa. El desconocimiento y falta de formación en materia de discapacidad fue identificado como uno de los mayores obstáculos para el aprendizaje e inclusión de estos estudiantes. Este profesorado consideraba que la formación era imprescindible y creía que le podría beneficiar, ya que, en muchas ocasiones, sentían presión social o temor a la hora de hacer frente a situaciones nuevas relacionadas con las necesidades de los estudiantes. La formación les podía llevar a sentirse seguros en relación a las realidades encontradas en las aulas.

Aquellos profesores en cuyas asignaturas estuvieron matriculados estudiantes con discapacidad, adquirieron experiencia sobre el tema y se sintieron sensibilizados, pero no supieron cómo atender sus necesidades. Por ello, consideraron esta capacitación como imprescindible.

Creemos que estamos preparados hasta que te enfrentas con el problema y te das cuenta que no, no estás preparado (Profesor 17).

Por otro lado, se creía necesario que el profesorado estuviera primero sensibilizado, y posteriormente se formase sobre este tema. Se justificó esta idea teniendo en cuenta que la formación no debería ser de carácter voluntaria, ya que es un medio que favorecía el cambio de actitudes para llegar a ser un buen profesional y así poder enseñar a todos los alumnos desde la diversidad, respetando la heterogeneidad.

Los docentes consideraban que era necesario saber qué hacer ante determinadas situaciones para no tener que actuar de manera improvisada. De ahí la necesidad de sentir mayor seguridad en el trato que ofrecían a los estudiantes y estar mejor preparados para dar respuestas adecuadas.

Entonces, tengo miedo a enfrentarme a casos que no sepa por dónde cogerlos y también a discriminar al alumno, tanto por defecto como por exceso (Profesor 13).

\section{Un profesorado sensibilizado}

Tras realizar la formación, los participantes destacaban que se sentían más seguros, ya que contaban con información sobre estos temas. Señalaron la toma de conciencia experimentada sobre la situación que atravesaban los estudiantes con discapacidad, además de la importancia que cobraba ofrecerles una respuesta educativa adecuada.
Tras su paso por el programa, los participantes identificaban con más énfasis la necesidad de formación en discapacidad y educación inclusiva, a pesar de la alta motivación de la que ya partían.

Indicaron que el programa había contribuido a desarrollar en mayor medida la empatía y la sensibilidad con este alumnado, y les motivó para prestar más atención a su actuación docente con los estudiantes con discapacidad:

...aprender a empatizar con el alumnado con discapacidad y sensibilizarnos de que podemos hacer más (Profesor 10).

La carrera de obstáculos que tienen que salvar muchos de estos estudiantes ha hecho que los participantes se replanteasen su labor docente. En definitiva, explicaron cómo tomaron un compromiso mayor para la mejora de los procesos de atención a los estudiantes tras conocer de cerca cómo es realmente su situación en la institución universitaria:

A veces ya no solo lo que aprendes en los contenidos, sino que ahora te haces preguntas que antes no te hacías, porque como tienes información que no... Ahora tengo unas dudas que antes no se me hubieran ocurrido, pero no lo veo como algo negativo, sino como algo positivo (Profesor 10).

\section{La necesidad de información sobre el alumnado con discapacidad}

El profesorado, según comentó antes de realizar la formación, no tenía suficiente información sobre los estudiantes con discapacidad. Desconocían la normativa que regulaba los derechos del alumnado, las obligaciones de los profesores con respecto a estos y los ajustes razonables que podían realizar. Además, no sabía que existía en la universidad una oficina de apoyo a la discapacidad, tanto para ayudar a los estudiantes como al profesorado.

Algunos de los argumentos que justificaban la importancia de la formación aludían a la obligación de atender a estos alumnos de acuerdo a la normativa universitaria, como el cumplimiento de los criterios de una calidad docente.

Porque la ley lo ampara, porque es un derecho del alumno que tienes delante y, por tanto, se convierte en una obligación para el que organiza el aula, que es el profesor (Profesor 2).

En este sentido, uno de los primeros temas que emergió era el de conocer la normativa universitaria en materia de discapacidad, algo que consideraban fundamental, pero que desconocían totalmente.

Yo la normativa, desconozco absolutamente qué dice la ley respecto a la universidad. Conozco la ley en Educación Primaria pero la de la universidad la desconozco absolutamente (Profesor 2). 
También, el profesorado señalaba como necesidad de formación conocer los servicios universitarios y al personal especializado en la universidad a los que podrían acudir para buscar apoyo, y aprender las pautas a aplicar para tratar estos casos con equidad.

Sin embargo, la mayor preocupación residía en cómo aplicar la ley. Eran conocedores de que la normativa podía aportar el "qué" hacer, pero no el "cómo".

\section{Un profesorado informado}

Una vez finalizado el programa de formación, los docentes valoraban saber que en la normativa se hacía explícito el derecho que tenían los estudiantes con discapacidad a que se realizaran las modificaciones necesarias en el currículo. El desconocimiento inicial de los aspectos normativos hacía que les surgiesen numerosas dudas a la hora de tomar decisiones acerca de cómo responder a las peticiones de los estudiantes. Una vez aprendidos, estos conocimientos les tranquilizaban, ya que sus actuaciones no dependían de su buena voluntad, sino que era un derecho reconocido legalmente para los estudiantes.

A mí me ha dado como seguridad, el saber que si llega un caso donde se requiera $X$ adaptaciones, eso está contemplado y se puede hacer (Profesor 13).

En este sentido, destacaban los contenidos que les habían permitido conocer la existencia del servicio de atención al alumnado con discapacidad, así como sus funciones y el asesoramiento y ayudas que ofrecía. Además, el descubrimiento de la normativa universitaria que regulaba los derechos y deberes en torno a este alumnado también era un aspecto muy relevante para el profesorado.

Pero sí, me ha dado recursos a la hora de saber... dónde puedo ir a preguntar, la unidad del SACU (Servicio de atención), que la verdad es que no tenía mucho conocimiento de ella (Profesor 5).

\section{¿En qué formarse? La formación práctica que necesita el profesorado}

Finalmente, los intereses de los participantes se centraban en adquirir conocimientos útiles y aplicables, más allá del aprendizaje de teoría y leyes. Cuando hablaban de conocimientos útiles hacían referencia a aspectos como los ajustes curriculares, en el proyecto docente, materiales, etc.

Vamos a imaginarnos que está este alumno aquí con estas características y ahora saca tu material y adáptalo, ¿cómo lo harías? Entonces, creo que habría que adquirir conceptos y temática teórica, pero después, práctica, practicar por si se nos da el caso, aprovechar los recursos que tenemos ahí y ponerlos en práctica (Profesor 13).
A los participantes les interesaba sobre todo aprender a realizar ajustes en la actividad docente. De entre éstos, los que más se repetían en el discurso de los docentes entrevistados eran aquellos referentes a adaptaciones en los tiempos y formatos de examen, en los materiales de estudio, en las actividades de aula o en las presentaciones con diapositivas.

...veamos, este Power Point... lo voy a adaptar para una discapacidad visual. Eso me parece formidable, y también las adaptaciones de los proyectos docentes, eso es lo que necesitamos precisamente (Profesor 3).

Por último, un aspecto controvertido para los docentes era la dificultad de aplicar las herramientas y competencias adquiridas a todo el alumnado con discapacidad, independientemente del caso concreto y estableciendo un proceso generalizado:

A mí me gustaría saber hacer frente, pero desde algo que llegue a ser normativo, es decir, que no sea algo especialmente 'como tengo este caso, y cómo me voy a enfrentar...', sino el cómo lo puedo incluir para que sea un alumno más (Profesor 5).

\section{Un profesorado formado}

En respuesta a estas necesidades iniciales, los participantes en el estudio adquirieron una serie de conocimientos, habilidades y herramientas que, según comentaban, les permitirían atender adecuadamente a las demandas de los estudiantes con discapacidad.

Un tema que creían podían resolver, pero que en muchos casos no se hacía, era el referido a las modificaciones del currículo. Consideraban que como docentes podían pensar estar preparados para hacerlo, pero la realidad era que no lo estaban.

Los profesores destacaron, además, los aprendizajes relacionados con la elaboración de materiales accesibles. Uno de los objetivos del programa de formación residía en capacitar a los docentes para que pudieran diseñar materiales adaptados. Por ejemplo, se formaba en cómo realizar un Power Point accesible. Los participantes valoraron positivamente este aprendizaje, indicando que el esfuerzo dedicado a esta tarea era una inversión para el futuro, puesto que se trataba de producir materiales que podían utilizarse en cursos posteriores y que beneficiaba a todo el alumnado:

Yo por ejemplo con el Power Point ya me ha quedado claro, la regla del 6 , y yo ya voy contando, no puedes poner más de 6 tal y cual (Profesor 13).

Los diferentes tipos ajustes a aplicar en los materiales se consideraron de gran utilidad, como el aumento del tamaño de letra en textos escritos, la utilización de recursos tecnológicos o las transcripciones de audios y/o vídeos. 
Por otro lado, identificaron una serie de medidas de "sentido común" de las que no eran conscientes antes de recibir la formación, ya que consistían en evitar detalles que se ponían en práctica de manera involuntaria en el aula. Destacaron algunas como no hablar dando la espalda al estudiante, facilitar que el alumno se sentara en lugares estratégicos y cercanos, no moverse en exceso o hablar de manera pausada. Al no tener en cuenta estos detalles, podían dificultar en gran medida el aprendizaje de los estudiantes con discapacidad, y esta formación les ayudó a poder identificarlos y reemplazarlos por respuestas más acertadas.

Yo los conocimientos de adaptación, desde el punto de vista profesional, creo que son todos importantísimos, o sea, los destacaría sobre todos. La capacidad que tengo desde la oralidad, cómo expresarse, cómo andar en la clase, cómo fomentar la inclusión social entre los diferentes alumnos... (Profesor 15).

En cuanto a la modificación de una asignatura para incluir al alumnado con discapacidad, también se podía apreciar un impacto del programa. Los participantes cambiaron sus concepciones desde una respuesta reactiva a proactiva. Es decir, si antes de la formación pensaban que las modificaciones necesarias en una asignatura debían ser realizadas una vez detectado el problema y comenzada la asignatura, después de ésta pensaban lo contrario. Los profesores entendían que sus proyectos docentes tenían que ser accesibles, dando cabida a todos los estudiantes y basándose en el DUA.

Lo que resume para mí lo aprendido en el curso y de aplicación práctica es la percepción de que no es el alumno con discapacidad el molde al que yo tengo que adaptar, hacer que mi asignatura encaje...Es, más bien al contrario, es mi asignatura la que tiene que estar preparada, diseñada desde el principio para ser un molde tan versátil, que permita que cualquier alumno, sean cuales sean sus circunstancias, encaje (Profesor 17).

Los principios del DUA fueron tomados por el profesorado como un concepto que englobaba todos los aprendizajes previos. Encontraban así la forma de realizar un diseño de la programación que atendiera a las necesidades de todos los estudiantes previamente a que éstas aparecieran en el aula, estando preparados para dar respuesta a todas ellas. Identificaron así la importancia de analizar todos los componentes del proyecto docente, adaptando o flexibilizando aquellos que pudieran suponer una barrera para algún estudiante.

Es esa adaptación del proyecto docente, atendiendo a las pautas que ofrece el DUA, la concreción práctica de todo lo aprendido en el programa, siendo uno de los conocimientos que los participantes valoraron como más valiosos, prácticos y necesarios en su día a día profesional.

\section{Discusión y conclusiones}

El profesorado que ha participado en este estudio reconocía, antes de recibir la formación, una serie de necesidades formativas y expresaba su deseo de recibir una formación en relación a cómo dar una respuesta inclusiva al alumnado con discapacidad. Otros trabajos han llegado a conclusiones similares, que coinciden en los mismos resultados y además, han estudiado cómo formar al profesorado universitario en materia de discapacidad (Debrand \& Salzberg, 2005; Healey, Jenkins, Leach, \& Roberts, 2001; Hockings \& cols., 2012). En algunos de los estudios realizados se concluye que la actitud del profesorado mejora una vez que éste se forma y cuenta con más experiencia sobre cómo responder a las necesidades de los estudiantes con discapacidad (Hong, 2015; Murray \& cols., 2011).

Además, los participantes de este estudio proponían qué contenidos serían esenciales para sentirse preparados y seguros para dar respuesta a las diversas necesidades del alumnado. Para este profesorado, al igual que en los trabajos de Cook y cols. (2009) y Gelbar y cols. (2015), sería fundamental incluir contenidos sobre servicios y recursos que facilita la universidad, además de normativas universitarias en materia de discapacidad. Pero más allá de este conocimiento legal, para el profesorado es imprescindible una formación práctica de cómo aplicar la normativa.

Este trabajo responde a la necesidad expresada en las investigaciones sobre universitarios con discapacidad, quienes destacaban que la principal barrera que se encontraban en sus estudios superiores era el profesorado y que este debía estar informado, formado y sensibilizado (Hopkins, 2011; Moswela \& Mukhopahdyay, 2011; Tinklin, Riddell, \& Wilson, 2004).

En diversos trabajos se ha concluido que la formación recibida impactó en la sensibilidad hacia los estudiantes con discapacidad y contribuyó a mejorar las actitudes del profesorado (Davies \& cols., 2013; Lomardi \& cols., 2011; Murray \& cols.,2011; Schelly, Davies, \& Spooner, 2011). En nuestro estudio se llega a un resultado similar y los profesores declaraban sentirse más motivados y sensibles hacia las necesidades estos estudiantes. El cambio de actitudes de los docentes es destacado en gran medida también por los propios estudiantes (Moriña, López-Gavira, \& Molina, 2016). Esta conclusión es muy interesante si se tiene en cuenta que para los estudiantes es importante el aspecto afectivo y emocional del docente y valoran su "aspecto humano".

Por otro lado, un profesorado informado no debe llevar a cabo actuaciones que dependen de su buena voluntad, sino que conoce que hay una normativa que regula las modificaciones que se pueden hacer del currículo y los derechos que tienen los estudiantes con discapacidad. Estar informado, por tanto, es un paso inicial que evitaría algunas de las barreras señaladas por los estudiantes en otras investigaciones (Fuller \& cols., 2004; Leyser \& cols, 2000).

Además, no es suficiente con estar informado y sensibilizado, también el profesorado debe estar formado. En este estudio los participantes destacaron que habían 
aprendido a saber cómo realizar modificaciones curriculares y cómo diseñar y desarrollar proyectos docentes desde los principios docentes del DUA.

En relación a la formación, también este profesorado resalta que las mejoras introducidas para los estudiantes con discapacidad benefician, no solo a estos, sino a todos los estudiantes. En los trabajos de otros autores se ha llegado a la misma conclusión (Gorard \& cols., 2006; Pliner \& Johnson, 2004).

Los resultados de este trabajo nos llevan a pensar que la formación del profesorado en materia de discapacidad y educación inclusiva es una pieza clave para aquellos sistemas universitarios que están interesados en construir procesos educativos inclusivos. Es, por tanto, necesario que se articulen políticas, procesos y acciones para que la respuesta a las necesidades de los estudiantes no se quede en la buena voluntad y se cuente con profesorado sensibilizado, informado y formado.

Por último, sería recomendable que se pusieran en marcha nuevas iniciativas de formación de profesorado en otras universidades. Además, otras líneas futuras de investigación podrían encaminarse al conocimiento de las buenas prácticas inclusivas que el profesorado lleva a cabo en las aulas universitarias, con el fin de compartir esas experiencias con el resto de docentes y dar a conocer los métodos y recursos educativos que están favoreciendo la inclusión de todos los estudiantes.

\section{Referencias}

Bell, S.; Devecchi, C.; Guckin, C.M.; Shevlin, M. (2017). Making the transition to post-secondary education: opportunities and challenges experienced by students with ASD in the Republic of Ireland. European Journal of Special Needs Education, 32(1), 5470. doi: $10.1080 / 08856257.2016 .1254972$

Bessant, J. (2012). 'Measuring Up'? Assessment and students with disabilities in the modern university. International Journal of Inclusive Education, 16(3), 265-281. doi: 10.1080/13603116.2010.489119

Black, R.D.; Weinberg, L.A.; Brodwin, M. G. (2014). Universal design for instruction and learning: A pilot study of faculty instructional methods and attitudes related to students with disabilities in higher education. Exceptionality Education International, 24(1), 48-64. Recuperado: 2 abr. 2017. Disponível: http://ir.lib.uwo.ca/eei/vol24/ iss $1 / 5$

Carballo, R. (2016). La formación del profesorado universitario: una perspectiva a través de la oferta formativa y los responsables de la formación. Deutschland: EAE.

Cook, L.; Rumrill, P.D.; Tankersley, M. (2009). Priorities and understanding of faculty members regarding college students with disabilities. International Journal of Teaching and Learning in Higher Education, 21(1), 84-96.
Cunningham, S. (2013). Teaching a diverse student body - a proposed tool for lecturers to self-evaluate their approach to inclusive teaching. Practice and Evidence of the Scholarship of Teaching and Learning in Higher Education, 8(1), 3-27. Recuperado: 2 abr. 2017. Disponível: http://eprints.mdx.ac.uk/11029/

Davies, P.L.; Schelly, C.L.; Spooner, C.L. (2013). Measuring the effectiveness of Universal Design for Learning intervention in postsecondary education. Journal of Postsecondary Education and Disability, 26(3), 195-220. Recuperado: 24 mar. 2017. Disponível: https://eric.ed.gov/?id=EJ1026883

Debrand, C.C.; Salzberg, C.L. (2005). A Validated Curriculum to Provide Training to Faculty regarding Students with Disabilities in Higher Education. Journal of Postsecondary Education and Disability, 18(1), 49-61. Recuperado: 6 fev. 2017. Disponível: https://eric.ed.gov/?id=EJ846380

Dotras, P.; Llinares, M.; López, P. (2008, abril). Propuesta de formación al profesorado en el contexto de la Uniersiad Pública. Trabajo presentado en el $\mathrm{V}$ Congreso Internacional de Psicología y Educación, Oviedo - España.

Fuller, M.; Healey, M.; Bradley, A.; Hall, T. (2004). Barriers to learning: a systematic study of the experience of disabled students in one university. Studies in Higher Education, 29(3), 303-318. doi: 10.1080/03075070410001682592

Garrison-Wade, D.F. (2012). Listening to Their Voices: Factors that Inhibit or Enhance Postsecondary Outcomes for Students' with Disabilities. International Journal of Special Education, 27(2), 113-125. Recuperado: 12 nov. 2016. Disponível: https://eric. ed.gov/?id=EJ982866

Gelbar, N.W.; Madaus, J.W.; Lombardi, A.; Faggella-Luby, M.; Dukes, L. (2015). College students with physical disabilities: common on campus, uncommon in the literature. Physical Disabilities: Education and Related Services, 34(2), 14-31. doi: 10.14434/ pders.v34i2.19224

Gorard, S.; Smith, E.;May, H.; Thomas, L.; Adnett, N.; Slack, K. (2006). Review of widening participation research: addressing the barriers to participation in higher education. Bristol: HEFCE.

Griffiths, S. (2010). Teaching for Inclusion in Higher Education: A Guide to Practice. Dublin: All Ireland Society for Higher Education.

Gunersel, A.B.; Etienne, M. (2014). The impact of a faculty training program on teaching conceptions and strategies. International Journal of Teaching and Learning in Higher Education, 26(3), 404-413. Recuperado: 9 jan. 2017. Disponível: https://eric. ed.gov/?id=EJ1061039

Hardy, I.; Woodcock, S. (2015). Inclusive education policies: discourses of difference, diversity and deficit. International Journal of Inclusive Education, 19(2), 141-164. doi: 10.1080/13603116.2014.908965 
Healey, M.; Jenkins, A.; Leach, J.; Roberts, C. (2001). Issues in Providing Learning Support for Disabled Students Undertaking Fieldwork and Related Activities. Gloucestershire: Geography Discipline Network.

Hockings, C.; Brett, P.; Terentjevs, M. (2012). Making a differenceinclusive learning and teaching in higher education through open educational resources. Distance Education, 33(2), 237-252. doi: 10.1080/01587919.2012.692066

Hong, B.S.S. (2015). Qualitative Analysis of the barriers college students with disabilities experiences in higher education. Journal of College Student Development, 56 (3), 209-226. doi: 10.1353/ csd.2015.0032

Hopkins, L. (2011). The path of least resistance: a voice-relational analysis of disabled students' experiences of discrimination in English universities. International Journal of Inclusive Education, 15, 711-727. doi: 10.1080/13603110903317684

Leyser, Y.; Vogel, S.; Wyland, S.; Brulle, A.; Sharoni, V.; Vogel, G. (2000). Students with disabilities in higher education: Perspectives of American and Israeli faculty members. International education, 29(2), 47. Recuperado: 16fev.2017. Disponível: http:// search.proquest.com/openview/57aa432795aae61ed918ffcc80fa 7c81/1?pq-origsite $=$ gscholar $\&$ cbl= 1818844

Lombardi, A. R.; Murray, C.; Gerdes, H. (2011). College faculty and inclusive instruction: Self-reported attitudes and actions pertaining to Universal Design. Journal of Diversity in Higher Education, 4(4), 250-261. doi: 10.1037/a0024961

Messiou, K.; Ainscow, M.; Echeita, G.; Goldrick, S.; Hope, M.; Paes, I.; ... Vitorino, T. (2016). Learning from differences: a strategy for teacher development in respect to student diversity. School Effectiveness and School Improvement, 27(1), 45-61. doi: 10.1080/09243453.2014.966726

Miles, M.B.; Huberman, A. M. (1994). Qualitative data analysis. Thousand Oaks, CA: Sage Publications.

Milic, M.; Dowling, M. (2015). Social support, the presence of barriers and ideas for the future from students with disabilities in the higher education system in Croatia. Disability \& Society, 30(4), 614-629. doi: 10.1080/09687599.2015.1037949

Moriña, A. (2017). Inclusive education in higher education: challenges and opportunities. European Journal of Special Needs Education, 32(1), 3-17. doi: 10.1080/08856257.2016.1254964

Moriña, A. (2018). Formación de profesorado para una educación inclusiva en la universidad. Madrid: Síntesis.

Moriña, A.; López-Gavira, R.; Molina, V.M. (2016). What if we could Imagine an Ideal University? Narratives by Students with Disabilities. International Journal of Disability, Development and Education, 64(4) 353--367. doi: 10.1080/1034912X.20161228856
Moswela, E.; Mukhopadhyay, S. (2011). Asking for too much? The voices of students with disabilities in Botswana. Disability \& Society, 26(3), 307-319. doi: 10.1080/09687599.2011.560414

Mullins, L.; Preyde, M. (2013). The lived experience of students with an invisible disability at a Canadian university. Disability \& Society, 28(2), 147-160. doi: 10.1080/09687599.2012.752127

Murray, M.; Lombardi, A.; Wren, C.T. (2011). The effects of disabilityfocused training on the attitudes and perceptions of university staff. Remedial and Special Education, 32(4), 290-300. doi: $10.1177 / 0741932510362188$

Pliner, S.M.; Johnson, J.R. (2004). Historical, theoretical, and foundational principles of universal instructional design in higher education. Equity \& Excellence in Education, 37(2), 105-113. doi: $10.1080 / 10665680490453913$

Redpath, J., Kearney, P., Nicholl, P., Mulvenna, M., Wallace, J.\& Martin, S. (2013). A qualitative study of the lived experiences of disabled post-transition students in higher education institutions in Northern Ireland. Studies in Higher Education, 38(9), 1334-1350. doi: 10.1080/03075079.2011.622746

Schelly, C.L.; Davies, P.L.; Spooner, C.L. (2011). Student Perceptions of Faculty Implementation of Universal Design for Learning. Journal of Postsecondary Education and Disability, 24(1), 17-30. Recuperado: 12 nov. 2016. Disponível: https://eric. ed.gov/?id=EJ941729

Simpson, A. (2002). The Teachability Project: Creating an Accessible Curriculum for Students with Disabilities. Planet, 6(1), 13-15. doi:10.11120/plan.2002.00060013

Stein, K.F. (2014). Experiences of College Students with Psychological Disabilities: The Impact of Perceptions of Faculty Characteristics on Academic Achievement. International Journal of Teaching and Learning in Higher Education, 26(1), 55-65. Recuperado: 16 fev. 2017. Disponível: https://eric.ed.gov/?id=EJ1043011

Strnadová, I.; Hájková, V.; Květoňová, L. (2015). Voices of university students with disabilities: inclusive education on the tertiary level - a reality or a distant dream? International Journal of Inclusive Education, 19(10), 1080-109. doi:10.1080/13603116.2015.10378 68

Tinklin, T.; Riddell, S.; Wilson, A. (2004). Policy and provision for disabled students in higher education in Scotland and England: The current state of play. Studies in Higher Education, 29(5), 637657. doi: 10.1080/0307507042000261599

Vickerman, P.; Blundell, M. (2010). Hearing the voices of disabled students in higher education. Disability \& Society, 25(1), 21-32. doi: 10.1080/09687590903363290

Yssel, N.; Pak, N.; Beilke, J. (2016) A Door Must Be Opened: Perceptions of Students with Disabilities in Higher Education. 
International Journal of Disability, Development and Education, 63(3), 384-394. doi: 10.1080/1034912X.2015.1123232

Este trabajo se enmarca dentro de un proyecto de investigación financiado por el Ministerio de Economía y Competitividad de España, "Caminando hacia la inclusión social y educativa en la universidad" (Ref. EDU2013-46303-R).
License information: This is an open-access article distributed under the terms of the Creative Commons Attribution License (type CC$\mathrm{BY}$ ), which permits unrestricted use, distribution and reproduction in any medium, provided the original article is properly cited.

Recebido em: 22/08/2017

Aprovado em: 11/12/2017

\section{Sobre os autores}

Anabel Moriña (anabelm@us.es)

Profesora Titular del Departamento de Didáctica y Organización Educativa de la Universidad de Sevilla. Su trayectoria profesional está vinculada a la educación inclusiva, exclusión educativa, formación del profesorado, discapacidad y Enseñaza Superior. Ha dirigido diversas investigaciones sobre Educación Superior y Discapacidad.

https://orcid.org/0000-0002-0852-7523

Rafael Carballo (rafcardel1@alum.us.es)

Estudiante de Doctorado en Educación y Licenciado en Pedagogía por la Universidad de Sevilla, con Máster en Psicología del Trabajo en la Universidad Complutense de Madrid. Profesor asociado en la Facultad de Humanidades y Ciencias Sociales de la Universidad Internacional Isabel I, España. Su actividad investigadora se desarrolla en un equipo de la Universidad de Sevilla dedicado a la inclusión de los estudiantes universitarios con discapacidad. Sus principales líneas de investigación se centran en la educación inclusiva, la atención al alumnado con discapacidad en la educación superior y la formación de profesorado universitario.

https://orcid.org/0000-0001-9591-7708 